\title{
CARACTERIZAÇÃo DE CIMENTO ODONTOLÓGICO OBTIDO A PARTIR DE UM VIDRO PREPARADO PELO MÉTODO DOS PRECURSORES POLIMÉRICOS
}

\author{
Marcio José Bertolini*, Maria Aparecida Zaghete, Rossano Gimenes e Carlos de Oliveira Paiva-Santos \\ Instituto de Química de Araraquara, Universidade Estadual Paulista, CP 355, 14801-970 Araraquara - SP \\ Regina Guenka Palma-Dibb \\ Faculdade de Odontologia de Ribeirão Preto, Universidade de São Paulo, Av. do Café, s/n, 14040-904 Ribeirão Preto - SP
}

Recebido em 27/7/04; aceito em 20/12/04; publicado na web em 13/4/05

\begin{abstract}
CHARACTERIZATION OF DENTAL CEMENT OBTAINED FROM A GLASS PREPARED BY THE POLYMERIC PRECURSOR METHOD. Glass ionomer cements are glass and polymer composite materials. These materials currently find use in dentistry. The purpose of this work is to obtain glass powders based on the composition $4.5 \mathrm{SiO}_{2}-3 \mathrm{Al}_{2} \mathrm{O}_{3}-2 \mathrm{CaO}$ to be used in dentistry. The powders were prepared by a chemical route at $700{ }^{\circ} \mathrm{C}$. The properties of glass ionomer cements obtained from powders prepared at $700{ }^{\circ} \mathrm{C}$ were studied. Diametral tensile strength and microhardness were evaluated for the experimental glass ionomer cements and a commercial material. It was concluded that the properties of experimental cements were similar to those of the commercial ones.
\end{abstract}

Keywords: cements; dental; glass.

\section{INTRODUÇÃO}

Cimento de ionômero de vidro (CIV) é um material compósito vidro/polímero, no qual as partículas de vidro têm função de material de preenchimento e são fonte de cátions para formação de ligações cruzadas com as cadeias poliméricas. Os cimentos são formados pela mistura de partículas vítreas com solução aquosa de ácidos poliméricos orgânicos (geralmente ácido poliacrílico) ${ }^{1,2}$. Quando as partículas de vidro do tipo cálcioaluminosilicato são misturadas com solução aquosa de ácido poliacrílico forma-se uma pasta, que pode ser facilmente manipulada pelo dentista e usada em várias aplicações odontológicas, como cimentação de dispositivos protéticos, forração de cavidades e nas restaurações ${ }^{3}$.

Os CIV adquirem presa inicial via reação de neutralização do tipo ácido/base entre os seus componentes. $\mathrm{O}$ processo envolve a hidrólise ácida das ligações $\mathrm{Si}-\mathrm{O}$ - Al da rede vítrea, resultando na liberação dos cátions $\mathrm{Ca}^{2+}$ e $\mathrm{Al}^{3+}$ e na formação de ácido ortosilícico, o qual se polimeriza formando sílica gel ${ }^{4,5}$. Os cátions extraídos da rede vítrea $\mathrm{Ca}^{2+}$ e $\mathrm{Al}^{3+}$ são quelados pelos grupos carboxílicos, presentes na estrutura polimérica do ácido orgânico, formando sais de poliacrilato e iniciando a "pega" da pasta vidro/ ácido por geleificação" ${ }^{1}$ Dessa maneira, o cimento obtido é constituído de partículas vítreas, com superfície revestida por sílica gel, e que estão presentes em uma matriz formada pelos sais de poliacrilato, os quais são responsáveis pelo endurecimento dos cimentos.

Os CIV apresentam certas propriedades, tais como adesão à estrutura dentária, valor do coeficiente de expansão térmico linear similar ao do dente e biocompatibilidade com os tecidos dentários ${ }^{6-8}$. Essas propriedades propiciam o uso desses cimentos em Dentística Restauradora como agentes cimentantes, para forramento de cavidades, preenchimento de dentes anteriores e, também, como cimentos ósseos ${ }^{9}$.

Os sistemas vítreos mais utilizados em Odontologia como formadores de cimentos de ionômero de vidro são os baseados no

\footnotetext{
*e-mail: marciobert@yahoo.com.br
}

sistema ternário $\mathrm{SiO}_{2}-\mathrm{Al}_{2} \mathrm{O}_{3}-\mathrm{CaO}^{2,4,5}$ e apresentam razão molar $\mathrm{Al}: \mathrm{Si}$ igual ou superior a 1:2. A partir desse sistema originaram-se outros mais complexos, pela inclusão de novos componentes para conferir ao cimento odontológico propriedades melhores. Por ex., a maioria dos pós contêm em suas formulações óxidos de $\mathrm{BaO}$ ou $\mathrm{SrO}$, modificadores ópticos, que conferem ao cimento um aspecto estético semelhante à estrutura dentária. Propriedades como resistência mecânica e adesão ao dente podem ser melhoradas pela adição de fluoreto de cálcio $\left(\mathrm{CaF}_{2}\right)$ e pentóxido de fósforo $\left(\mathrm{P}_{2} \mathrm{O}_{5}\right)$, respectivamente, aos sistemas vítreos ${ }^{3}$.

Esses sistemas vítreos são preparados via fusão de mistura de óxidos ${ }^{5}$. Esse processo apresenta alguns inconvenientes, como por ex., dificuldade no controle da composição e homogeneidade química, além de usar temperaturas na faixa de 1100 a $1400{ }^{\circ} \mathrm{C}$ (dependendo da composição). Em virtude, desses problemas, outros métodos de preparação de materiais vítreos têm sido propostos, como o processo sol-gel ${ }^{10}$ e o método dos precursores poliméricos ${ }^{11}$. $\mathrm{O}$ uso desses processos traz algumas vantagens em relação à fusão da mistura de óxidos, como melhor controle da composição, melhor homogeneidade química, melhor controle morfológico, maior reatividade superficial e menor temperatura de síntese ${ }^{10}$. Ambos os métodos utilizam alcoóxidos e/ou precursores inorgânicos como nitratos, cloretos, carbonatos, etc.

O método dos precursores poliméricos ou processo Pechini consiste na formação de quelatos entre os cátions metálicos e ácidos $\alpha$-hidroxicarboxílicos, por ex., o ácido cítrico, e posterior poliesterificação sob aquecimento com álcool poliidroxílico (etilenoglicol). Com as reações desenvolvidas nesse processo, obtém-se uma resina polimérica, a qual possui os cátions metálicos uniformemente distribuídos. Em função da estabilidade das ligações do complexo metal - oxigênio e da coordenação tetraédrica dos átomos de $\mathrm{Si}$ e $\mathrm{Al}$ essa resina pode ser calcinada, em baixas temperaturas, para produzir óxidos cristalinos e vidros com partículas finas e composição quimicamente controlada.

Em recente busca na literatura, encontraram-se vários tipos de compostos preparados pelo método dos precursores poliméricos para uma série de aplicações, mas nenhuma informação foi encontrada a respeito de sistemas vítreos preparados por esse processo. 
Dessa forma, o objetivo dessa pesquisa é preparar pós referentes ao sistema ternário $\mathrm{SiO}_{2}-\mathrm{Al}_{2} \mathrm{O}_{3}-\mathrm{CaO}$ (a base dos vidros usados na preparação dos cimentos de ionômeros de vidro) pelo método dos precursores poliméricos, visando aplicações odontológicas.

\section{PARTE EXPERIMENTAL}

\section{Preparação do sistema $4,5 \mathrm{SiO}_{2}-3 \mathrm{Al}_{2} \mathrm{O}_{3}-2 \mathrm{CaO}$ pelo método} dos precursores poliméricos

Os reagentes usados para preparar os pós do sistema: $4,5 \mathrm{SiO}_{2}-$ $3 \mathrm{Al}_{2} \mathrm{O}_{3}-2 \mathrm{CaO}$ foram tetraetilortosilicato (TEOS), nitrato de alumínio, citrato de cálcio, ácido cítrico e etilenoglicol.

Primeiramente, foram preparadas as soluções de citrato de silício e citrato de alumínio, separadamente. A solução de citrato de silício foi preparada pela reação entre alcoóxido (TEOS) e ácido cítrico sob agitação e aquecimento constantes. A solução de citrato de alumínio foi obtida pela solubilização do sal precursor (nitrato de alumínio) na solução aquosa de ácido cítrico. Posteriormente, essas soluções foram misturadas e homogeneizadas (solução A).

Em seguida, solução de citrato de cálcio e etilenoglicol foi adicionada à solução $\mathrm{A}$, sob agitação e aquecimento constantes. Desta maneira obteve-se uma resina polimérica que foi tratada termicamente até $400{ }^{\circ} \mathrm{C}$, em forno tipo mufla. Após o tratamento térmico a resina foi triturada em almofariz de ágata. Depois dessa etapa, os pós foram tratados termicamente a $500{ }^{\circ} \mathrm{C}$ para eliminação da maior parte do material orgânico e, depois, calcinados a $700{ }^{\circ} \mathrm{C}$ por $4 \mathrm{~h}$.

\section{Preparação dos cimentos de ionômeros de vidro (CIV)}

Corpos de prova dos CIV experimentais foram preparados pela mistura dos pós preparados a $700^{\circ} \mathrm{C}(15-20 \mu \mathrm{m})$ com uma solução aquosa de ácido poliacrílico (45-50\% massa/massa), usando razão massa/massa de pó:líquido de 1:1. Os corpos de prova do CIV comercial foram preparados com razão de 2:1 (recomendada pelo fabricante).

\section{Caracterização do sistema preparado}

Para confirmar o estado amorfo, o sistema calcinado a $700{ }^{\circ} \mathrm{C}$ foi caracterizado por difratometria de raios $\mathrm{X}$ usando radiação $\mathrm{K} \alpha$ do $\mathrm{Cu}$ no equipamento Rint 2000 com ânodo rotatório de $\mathrm{Cu}$ da marca Rigaku.

A curva DTA (Análise Térmica Diferencial) foi obtida usando $20 \mathrm{mg}$ de amostra, razão de aquecimento de $10{ }^{\circ} \mathrm{C} / \mathrm{min}$ e atmosfera de $\mathrm{N}_{2}$ no equipamento da TA Instruments modelo 1600 .

Ressonância Magnética Nuclear de ${ }^{29} \mathrm{Si}$ e ${ }^{27} \mathrm{Al}$ foram usadas para investigar os aspectos estruturais do sistema preparado e de um pó comercial. Os espectros de RMN foram feitos em um Espectrômetro INOVA 300 Varian. Os espectros de ${ }^{29} \mathrm{Si}-\mathrm{MAS}$ ("Magic Angle Spinning") (59,5MHz) foram obtidos com rotor de Zircônio usando $\pi / 2$ pulsos de $45 \mathrm{~s}$ tendo como referência o tetrametilsilano (TMS). Os espectros de ${ }^{27} \mathrm{Al}-\operatorname{MAS}(78,15 \mathrm{MHz})$ foram obtidos com rotor de $\mathrm{Si}_{3} \mathrm{~N}_{4}$ com $\pi / 6$ pulsos de $2 \mu \mathrm{s}$ e $\mathrm{Al}\left(\mathrm{H}_{2} \mathrm{O}\right)_{6}^{3+}$ como referência.

\section{Caracterização dos cimentos de ionômero de vidro preparados}

Os cimentos foram caracterizados por FTIR após $24 \mathrm{~h}$ da manipulação. Para a obtenção dos espectros, os corpos de prova foram triturados e misturados com $\mathrm{KBr}$ e prensados na forma de pastilhas. Os espectros de FTIR foram obtidos no Espectrofotômetro Nicolet modelo Impact 400.
Ensaios de microdureza foram realizados no aparelho HMV Microhardeness Tester da marca Shimadzu. Nesses ensaios o número Knoop (KHN) foi determinado com base nas cinco endentações realizadas na superfície de cada corpo de prova. Essas endentações foram realizadas em cinco corpos de prova, imediamente após 10 min e 24 h da manipulação dos cimentos.

Os ensaios mecânicos foram feitos em corpos de prova com dimensões $2 \mathrm{~mm}$ x $4 \mathrm{~mm}$, após $24 \mathrm{~h}$ da manipulação. Nesses ensaios mecânicos oito corpos de prova foram preparados referentes aos CIV experimental e ao CIV comercial nas razões pó:líquido de $1: 1,0,66: 1$ e $2: 1$. As medidas foram realizadas no equipamento MTS -810 - Materials Test System.

\section{RESULTADOS E DISCUSSÃO}

\section{Caracterização do sistema preparado por processo químico}

O difratograma de raios $\mathrm{X}$, Figura 1, obtido para o pó após calcinação a $700{ }^{\circ} \mathrm{C}$ por $4 \mathrm{~h}$, não exibe picos referentes a qualquer fase cristalina, apenas um halo indicando estrutura amorfa, similar aos vidros.

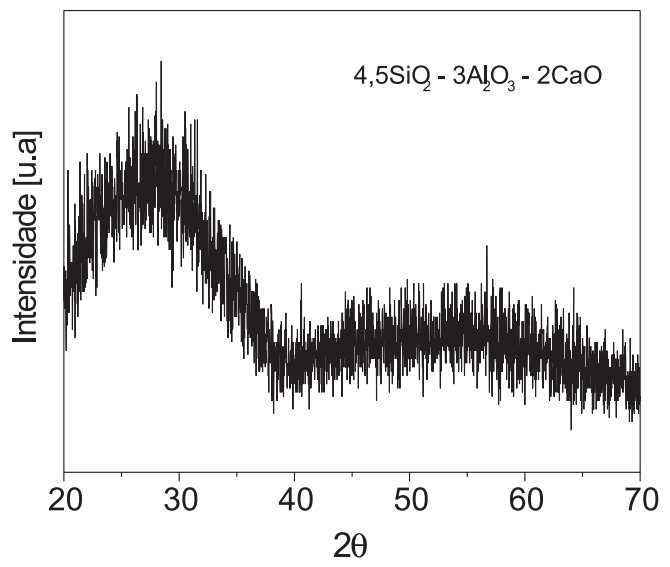

Figura 1. Difratograma de raios $X$ do sistema calcinado a $700{ }^{\circ} \mathrm{C} / 4 \mathrm{~h}$

Visando confirmar as características vítreas do material calcinado fez-se, também, a caracterização por Análise Térmica Diferencial (DTA), pois por esta técnica pode-se constatar a presença de temperaturas típicas dos vidros, tais como temperatura de transição vítrea $\left(\mathrm{T}_{\mathrm{g}}\right)$ e temperatura de cristalização $\left(\mathrm{T}_{\mathrm{c}}\right)$.

Na curva de DTA, Figura 2, nota-se que há uma mudança da linha base em aproximadamente $890{ }^{\circ} \mathrm{C}$, a qual pode ser associada à presença da temperatura de transição vítrea $\left(\mathrm{T}_{\mathrm{g}}\right)$. Observa-se também um pico exotérmico a $1000{ }^{\circ} \mathrm{C}$, o qual pode ser relacionado à temperatura de cristalização do vidro $\left(\mathrm{T}_{c}\right)$. Estes dois resultados permitem afirmar que o sistema amorfo obtido a $700{ }^{\circ} \mathrm{C}$ é um vidro.

A caracterização estrutural do sistema vítreo preparado foi feita por análises de RMN MAS de ${ }^{29} \mathrm{Si}$ e ${ }^{27} \mathrm{Al}$, fez-se as mesmas análises para um sistema comercial, com a finalidade de se ter um parâmetro de comparação. Os dois sistemas apresentaram espectros de RMN MAS ${ }^{29} \mathrm{Si}$ semelhantes. No espectro do material comercial observa-se um pico localizado em $-92,7$ ppm e para o sistema preparado neste trabalho, um pico em $-90,9$ ppm, Figura 3. Os valores de deslocamento obtidos, nessa faixa, são atribuídos a átomos de silício com estrutura $\mathrm{Q}_{3}{ }^{12,13}$, a qual se refere às ligações Si---O, com um átomo de oxigênio não ligado (ONL). Deste modo, pode-se inferir que a rede vítrea do sistema preparado é similar à do material comercial, onde os átomos de Si também possuem es- 


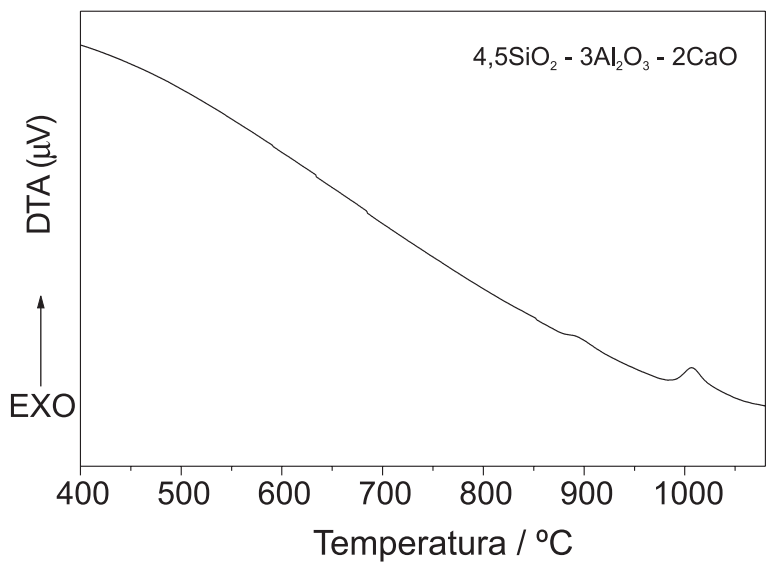

Figura 2. Curva de DTA do sistema $4,5 \mathrm{SiO}_{2}-3 \mathrm{Al}_{2} \mathrm{O}_{3}-2 \mathrm{CaO}$ obtida usando $20 \mathrm{mg}$ de amostra, razão de aquecimento de $10^{\circ} \mathrm{C} / \mathrm{min}$ e atmosfera de $\mathrm{N}_{2}$

trutura $\mathrm{Q}_{3}$. Essa estrutura é referente ao tetraedro $\left[\mathrm{SiO}_{4}\right]$ que forma a rede vítrea desses materiais.

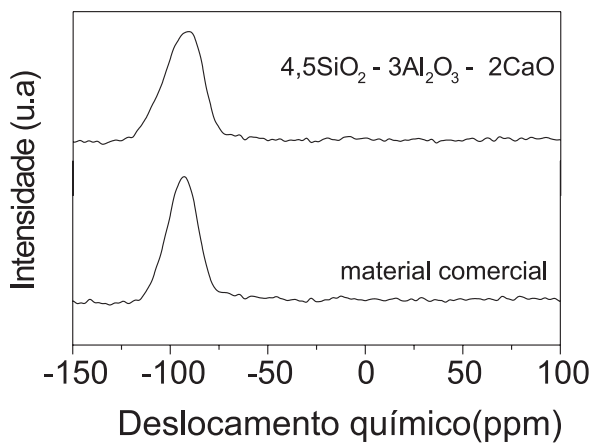

Figura 3. Espectros de RMN - MAS de ${ }^{29} \mathrm{Si}$ referentes ao sistema 4,5 $\mathrm{SiO}_{2}$ $3 \mathrm{Al}_{2} \mathrm{O}_{3}-2 \mathrm{CaO}$ e ao material comercial

Outro ponto importante a ser investigado é a coordenação dos átomos de $\mathrm{Al}$ na rede vítrea desses materiais. Quando os átomos de $\mathrm{Al}$ estão coordenados tetraedricamente $\mathrm{Al}^{[4]}$ os deslocamentos aparecem na faixa de 55 a $80 \mathrm{ppm}$, enquanto que se os átomos de $\mathrm{Al}$ estiverem octaedricamente coordenados, $\mathrm{Al}^{[6]}$, os deslocamentos se localizam na faixa de -10 a $10 \mathrm{ppm}^{14}$.

Nos espectros de RMN MAS de ${ }^{27} \mathrm{Al}$ obtidos para o sistema vítreo preparado neste trabalho e o material comercial, Figura 4, nota-se que existem dois picos indicativos de que os átomos de $\mathrm{Al}$ estão em dois estados diferentes de coordenação na rede vítrea, possivelmente como $\mathrm{Al}^{[4]} \mathrm{e} \mathrm{Al}^{[6]}$. Nos espectros apresentados na Figura 4, os picos referentes aos átomos de $\mathrm{Al}^{[4]}$ estão localizados em 54 ppm para o pó comercial e a 65 ppm para o sistema preparado neste trabalho. Os sinais referentes aos átomos de $\mathrm{Al}^{[6]}$ octaedricamente coordenados aparecem em -5,5 ppm para o pó comercial e em 0 ppm para o sistema preparado neste trabalho. Como reportado na literatura, a rede vítrea deve ser formada por tetraedros de $\left[\mathrm{SiO}_{4}\right]$ e de $\left[\mathrm{AlO}_{4}\right]$ ligados entre si. Esta é uma das condições para que a rede do sistema $\mathrm{SiO}_{2}-\mathrm{Al}_{2} \mathrm{O}_{3}-\mathrm{CaO}$ se torne susceptível ao ataque ácido para obtenção dos CIV. Quando os íons $\mathrm{Al}^{3+}$ substituem os íons $\mathrm{Si}^{4+}$, na rede vítrea, esta adquire uma carga negativa que é balanceada pela carga positiva de cátions modificadores como $\mathrm{Ca}^{2+}$ presentes na composição do vidro. $\mathrm{O}$ átomo de oxigênio que liga os tetraedros de alumínio e silício, bem como os átomos de oxigênio "não ligados" tornam a rede vítrea susceptível ao ataque ácido. Portanto, a presença desses sítios, na rede vítrea, é necessária para que esta possa ser vulnerável ao ataque ácido e formar os cimentos de ionômeros de vidro. As medidas de RMN indicaram que esses sítios estão presentes nas estruturas dos dois vidros analisados, portanto, pode-se inferir que a rede vítrea do material preparado neste trabalho é similar à do pó comercial, com átomos de $\mathrm{Si}\left(\mathrm{Q}_{3}\right)$ e átomos de alumínio tetraedrica e octaedricamente coordenados.

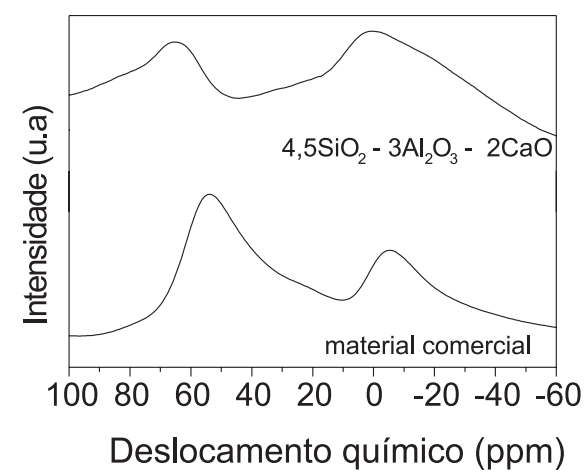

Figura 4. Espectros de $\mathrm{RMN}-\mathrm{MAS}$ de ${ }^{27} \mathrm{Al}$ referentes ao sistema $4,5 \mathrm{SiO}_{2}-$ $3 \mathrm{Al}_{2} \mathrm{O}_{3}-2 \mathrm{CaO}$ e ao material comercial

Considerando os resultados obtidos pode-se dizer que o vidro preparado será reativo frente ao ataque ácido e, portanto, tem potencial para ser utilizado como pó precursor dos cimentos de ionômero de vidro.

\section{Caracterização dos cimentos de ionômero de vidro (CIV)}

Espectroscopia vibracional na região do infravermelho (FTIR)

FTIR foi usado para investigar a reação de presa dos CIV. Os espectros foram obtidos após $24 \mathrm{~h}$ da manipulação dos cimentos e estão ilustrados na Figura 5. Nota-se a presença de bandas a $1464 \mathrm{e}$ $1648 \mathrm{~cm}^{-1}$, para o CIV comercial, e bandas a 1466 e $1646 \mathrm{~cm}^{-1}$, para o CIV obtido neste trabalho, as quais podem ser atribuídas aos estiramentos simétrico e assimétrico da ligação COO-Al. Isto confirma que as ligações $\mathrm{Si}-\mathrm{O}-\mathrm{Al}$ da rede vítrea foram hidrolisadas pelo ácido poliacrílico. De acordo com a literatura ${ }^{15}$, as bandas referentes ao estiramento das ligações $\mathrm{C}-\mathrm{O}$ e $\mathrm{C}=\mathrm{O}$ do ácido poliacrílico situam-se em 1250 e $1710 \mathrm{~cm}^{-1}$. Após a reação de neutralização, estas são progressivamente substituídas pelas bandas referentes ao

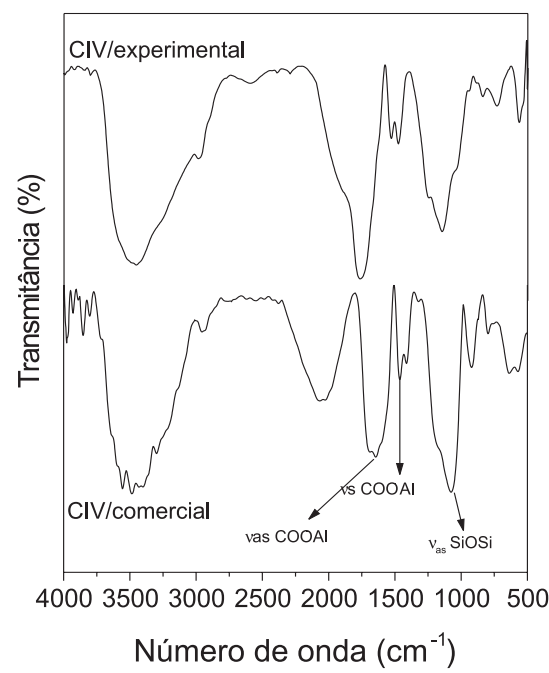

Figura 5. Espectros de FTIR dos CIV experimental e comercial após $24 \mathrm{hda}$ manipulação 
Tabela 1. Valores obtidos nos ensaios de resistência à tração diametral para os CIV experimental e comercial

\begin{tabular}{ccc}
\hline $\begin{array}{c}\text { CIV Comercial } \\
\text { Razão pó: líquido }(2: 1)\end{array}$ & $\begin{array}{c}\text { CIV Experimental } \\
\text { Razão pó: líquido }(1: 1)\end{array}$ & $\begin{array}{c}\text { CIV Experimental } \\
\text { Razão pó: líquido }(0,66: 1)\end{array}$ \\
\hline $11,96 \pm 3,3 \mathrm{MPa}$ & $9,31 \pm 2,11 \mathrm{MPa}$ & $6,27 \pm 0,92 \mathrm{MPa}$ \\
\hline
\end{tabular}

Tabela 2. Valores de microdureza Knoop (KHN) obtidos após 10 min e 24 h da manipulação dos CIV experimental e comercial

\begin{tabular}{lccc}
\hline Cimentos & $\begin{array}{c}\text { Razãopó:líquido } \\
\text { (massa/massa) }\end{array}$ & $\begin{array}{c}\text { Microdureza/10 min } \\
(\mathrm{KHN})\end{array}$ & $\begin{array}{c}\text { Microdureza/24 h } \\
(\mathrm{KHN})\end{array}$ \\
\hline CIV comercial & $2: 1$ & $5,75 \pm 0,55$ & $16,42 \pm 3,96$ \\
CIV experimental & $1: 1$ & $5,60 \pm 0,73$ & $18,78 \pm 5,3$ \\
\hline
\end{tabular}

poliacrilato de alumínio formado pela complexação dos íons $\mathrm{Al}^{3+}$, liberados da rede vítrea, com o ácido poliacrílico.

As bandas localizadas nas regiões de 3500 e $1600 \mathrm{~cm}^{-1}$ são atribuídas ao estiramento da ligação $\mathrm{O}-\mathrm{H}$ e à deformação angular da ligação $\mathrm{H}-\mathrm{O}-\mathrm{H}$, referentes aos modos vibracionais da água. $\mathrm{Na}$ faixa de 1100-1000 $\mathrm{cm}^{-1}$ encontra-se uma banda intensa referente ao estiramento assimétrico das ligações $\mathrm{Si}-\mathrm{O}$ da rede vítrea. As bandas localizadas na região de 720 a $700 \mathrm{~cm}^{-1}$ são atribuídas ao estiramento simétrico das ligações $\mathrm{Si}-\mathrm{O}-\mathrm{Si}$, no tetraedro $\mathrm{SiO}_{4}$, e as bandas localizadas em 460 a $400 \mathrm{~cm}^{-1}$ à deformação angular das ligações Si-O-Si, Al-O-Al ou Al-O-Si ${ }^{16}$.

\section{Resistência à tração diametral}

Ensaios de resistência à tração diametral são indicados para caracterizar os CIV usados como agentes cimentantes. Esses ensaios foram comparativos entre o CIV preparado a partir do vidro $4,5 \mathrm{SiO}_{2}-3 \mathrm{Al}_{2} \mathrm{O}_{3}-2 \mathrm{CaO}$ e o CIV comercial indicado para cimentação. A Tabela 1 mostra os resultados obtidos nesses testes.

Para esses testes foram preparados corpos de prova do CIV experimental com diferentes razões pó:líquido, visando obter uma pasta com boa trabalhabilidade. Utilizando razão pó:líquido de 1:1 ou de 0,66:1 foi possível obter uma pasta com consistência similar à do CIV comercial. No entanto, não foi possível obter corpos de prova usando a razão 2:1 igual ao CIV comercial, pois a pasta preparada com essa razão não apresentou boa trabalhabilidade. Como mostrado na Tabela 1, a razão pó:líquido influencia os resultados de resistência à tração diametral, pois com o decréscimo da razão de 1:1 para $0,66: 1$ o valor médio diminuiu de 9,31 MPa para 6,27 $\mathrm{MPa}$. Os corpos de prova do CIV comercial preparados com uma razão pó:líquido de 2:1 tiveram um valor médio de resistência à tração diametral maior que os do CIV experimental. No entanto, deve-se considerar que a maior razão pó:liquido utilizada para o CIV experimental foi de 1:1 e pela análise dos resultados obtidos, se fosse possível usar a razão de 2:1 provavelmente o valor da resistência estaria próximo ao valor obtido para o CIV comercial.

\section{Ensaios de microdureza}

Com o intuito de avaliar a "pega", tempo de presa inicial, do CIV experimental e do comercial realizaram-se medidas de microdureza Knoop para os cimentos após 10 min e 24 h da manipulação. A Tabela 2 ilustra os resultados obtidos nesses testes.

Observa-se que os valores de microdureza $(\mathrm{KNH})$ obtidos para os dois cimentos são semelhantes, tanto após 10 min quanto após $24 \mathrm{~h}$. Isto indica indiretamente que os tempos de presa inicial e após 24 h são próximos. Pode-se dizer também, que o CIV experimental mesmo preparado a partir de uma razão inferior à do CIV comercial apresenta reatividade frente ao ácido poliacrílico em um intervalo de tempo similar ao CIV comercial Outra observação importante a ser feita é que o tempo inicial de presa do CIV experimental é menor que $10 \mathrm{~min}$, o que atende às exigências feitas aos cimentos comerciais.

\section{CONCLUSÃO}

$\mathrm{O}$ método dos precursores poliméricos permitiu a preparação do sistema vítreo $\mathrm{SiO}_{2}-\mathrm{Al}_{2} \mathrm{O}_{3}-\mathrm{CaO}$ a $700{ }^{\circ} \mathrm{C}$.

Os cimentos preparados a partir do vidro obtido pelo processo químico apresentaram características como trabalhabilidade e tempo de presa similares ao CIV comercial.

\section{AGRADECIMENTOS}

À Fundação de Amparo à Pesquisa do Estado de São Paulo (FAPESP) e ao Conselho de Desenvolvimento Científico e Tecnológico $(\mathrm{CNPq})$ pelas bolsas de doutorado concedidas e pelo apoio financeiro para o desenvolvimento dessa pesquisa.

\section{REFERÊNCIAS}

1. Hurrell-Gillingham, K.; Reaney, I. M.; Miller, C. A.; Crawford, A.; Hatton, P. V.; Biomaterials 2003, 24, 3153.

2. Culbertson, B. M.; Prog. Polym. Sci. 2001, 26, 577.

3. Griffin, S. G.; Hill, R. G.; Biomaterials 1999, 20, 579.

4. Nicholson, J. W.; Biomaterials 1998, 19, 485.

5. Wilson, A. D.; Crisp, S.; Prosser, H. J.; Lewis, B. G.; Merson, S. A.; Ind. Eng. Chem. Prod. Res. Dev. 1980, 19, 263.

6. Sasanaluckit, P.; Albustany, K. R.; Doherty, P. J.; Williams, D. F.; Biomaterials 1993, 14, 906.

7. Stamboulis, A.; Law, R. V.; Hill, R. G.; Biomaterials 2004, 25, 3907.

8. Griffin, S. G.; Hill, R. G.; Biomaterials 2000, 21, 399.

9. Kenny, S. M.; Buggy, M.; J. Mater. Sci.: Mater. Med. 2003, 14, 923.

10. Taira, M.; Yamaki, M.; J. Mater. Sci.: Mater. Med. 1995, 6, 197.

11. Pechini, M.; US pat. 3,330,697 1967.

12. Schneider, J.; Mastelaro, V. R.; Panepucci, H.; Zanotto, E. D.; J. Non-Cryst. Solids 2000, 273, 8.

13. Schmucker, M.; Mackenzie, K. J. D.; Schneider, H.; Meinhold, R.; J. NonCryst. Solids 1997, 217, 99.

14. Mozgawa, W.; Fojud, Z.; Handke, M.; Jurga, S.; J. Mol. Struct. 2002, 614, 281.

15. Young, A. M.; Biomaterials 2002, 23, 3289.

16. Hwa, L. G.; Hwang, S. L.; Liu, L. C.; J. Non-Cryst. Solids 1998, $238,193$. 